\title{
Metal-Organic Framework Thin Films Composed of Free-Standing Acicular Nanorods Exhibiting Reversible Electrochromism
}

Chung-Wei Kung, ${ }^{\dagger}$ Timothy Chiaan Wang, ${ }^{\dagger}$ Joseph E. Mondloch, ${ }^{\dagger}$ David Fairen-Jimenez, ${ }^{\ddagger}$ Daniel M. Gardner, ${ }^{\dagger}$ Wojciech Bury, ${ }^{\dagger},{ }^{\circ}$ Jordan Matthew Klingsporn, ${ }^{\dagger}$ Jonathan C. Barnes, ${ }^{\dagger}$ Richard Van Duyne, ${ }^{\dagger}$ J. Fraser Stoddart, ${ }^{\dagger}$ Michael R. Wasielewski, ${ }^{\dagger}$ Omar K. Farha, ${ }^{*}{ }^{\dagger}$ and Joseph T. Hupp ${ }^{* \dagger}$

${ }^{\dagger}$ Department of Chemistry, Northwestern University, 2145 Sheridan Road, Evanston, Illinois 60208, United States

${ }^{\ddagger}$ Department of Chemical Engineering and Biotechnology, University of Cambridge, Pembroke Street, Cambridge CB2 3RA, United Kingdom

${ }^{\S}$ Department of Chemistry, Warsaw University of Technology, Noakowskiego 3, 00-664 Warsaw, Poland

\section{Supporting Information}

ABSTRACT: A uniform and crack-free metal-organic framework (MOF) thin film composed of free-standing acicular nanorods was grown on a transparent conducting glass substrate. The MOF thin film exhibits electrochromic switching between yellow and deep blue by means of a one-electron redox reaction at its pyrene-based linkers. The rigid MOF stabilizes the radical cations of the pyrene linkers at positive applied potential, resulting in the reversible color change of the MOF film. The regular and uniform channels of the MOF allow ions to migrate through the entire film. The MOF thin film thus exhibits a remarkable color change and rapid switching rate.

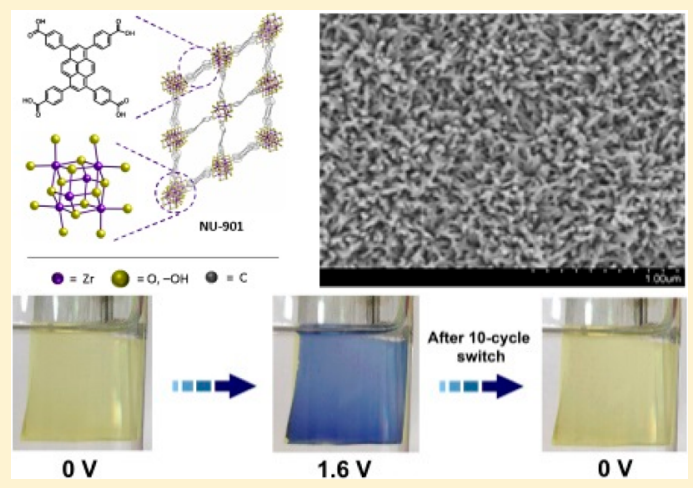

KEYWORDS: electrochromism, metal-organic frameworks, one-dimensional nanorods, pyrene

\section{INTRODUCTION}

Electrochromic materials exhibit reversible and persistent color change by means of redox reactions. ${ }^{1}$ These materials have attracted much attention over the last few decades because they can be used as smart windows, display devices, vehicle sunroofs, and antiglare mirrors for cars. ${ }^{2-4}$ Recently, electrochromic thin films have come to the forefront as an attractive alternative to soluble electrochromic materials since they show faster switching rates and optical memory. ${ }^{5}$ Metal oxides, ${ }^{6,7}$ Prussian blue and its analogues, ${ }^{8,9}$ and conducting polymers ${ }^{2,10}$ have all found application as electrochromic thin films. Since it is imperative for these materials that ions be transported into and out of the film during the redox chemistry so as to maintain electroneutrality, ${ }^{11}$ faster ion transport should directly enhance the switching rate of electrochromic thin films. Thus, uniform and tunable porosity is an attractive feature that enables facile ion transport and ensures that the entire film is redox-active.

Metal-organic frameworks (MOFs) are a class of porous materials constructed from metal-based nodes and organic linkers. ${ }^{12-14}$ Their high permanent porosity, ${ }^{15}$ nanostructured pores, and tunable pore compositions have led to their consideration for a remarkably broad range of applications, including gas storage, ${ }^{16}$ chemical separations, ${ }^{17}$ catalysis, ${ }^{18,19}$ and chemical sensing. ${ }^{20}$ Given these exciting properties, MOFs would seem to be attractive candidates for electrochromic thin films. ${ }^{21}$ The first fundamental study of the electrochromism in flat MOF thin films containing naphthalene diimide-based linkers has been recently reported. ${ }^{22}$

We reasoned that nanostructured MOFs might well exhibit exceptional switching rates; it is well-known that materials with at least one dimension at the nanoscale, i.e., less than $30 \mathrm{~nm}$, can exhibit unique physical properties compared to their bulk counterparts. ${ }^{23}$ Among the various known nanostructures, onedimensional (1D) nanostructures have become the focus of intensive research because of their typically superior electron and thermal transport properties. ${ }^{24}$ Several MOF 1D nanostructures have been synthesized, including nanowires, ${ }^{25,26}$ nanotubes, ${ }^{27}$ and nanorods. ${ }^{28-31}$ However, these nanostructures have largely been prepared as crystalline powders. Much rarer are thin-film 1D nanostructured MOFs prepared on a substrate. $^{32-34}$ In light of electrochemical applications, fabrication of MOF thin films on conducting substrates is necessary. Furthermore, 1D nanostructures of MOFs arranged on a substrate are expected to exhibit comparatively facile

Received: November 10, 2013

Revised: December 3, 2013

Published: December 13, 2013 
charge transport along the axial direction through the 1D nanostructures to the substrate. ${ }^{35}$ However, to date, there are no studies reporting the electrochromism of MOF 1D nanostructures.

In this study, thin films composed of $1 \mathrm{D}$ free-standing acicular nanorods of MOF were grown on transparent fluorinedoped tin oxide (FTO) conducting glass platforms. A MOF constructed from Zr-nodes and TBAPy linkers (NU-901, vide infra; $\mathrm{H}_{4}$ TBAPy $=1,3,6,8$-tetrakis $(p$-benzoic acid $)$ pyrene $\left.{ }^{36}\right)$ was selected (Figure 1a). The electrochromic behavior occurs by

\section{a}

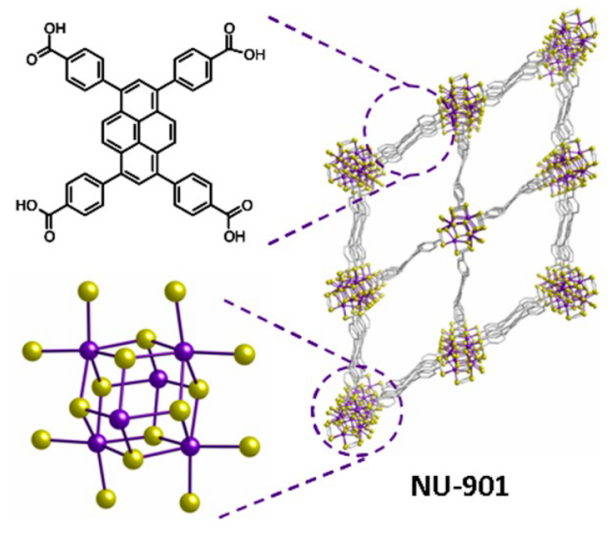

$\Theta=\mathrm{Zr} \quad \Theta=\mathrm{O},-\mathrm{OH} \quad \mathrm{O}=\mathrm{C}$

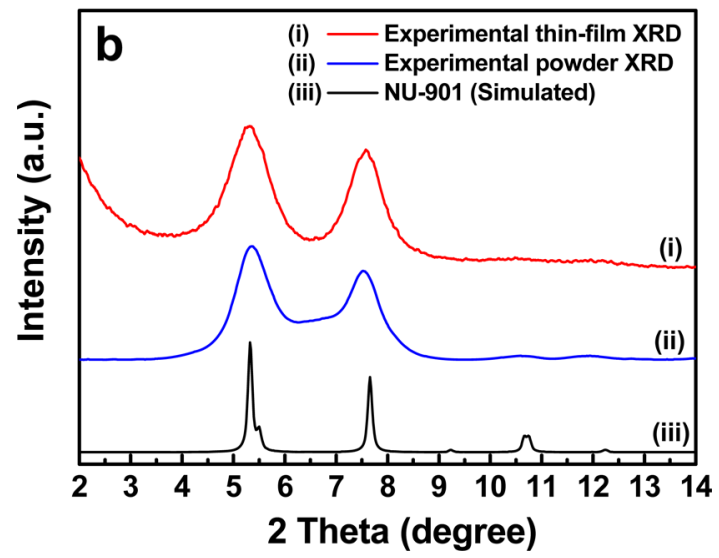

Figure 1. (a) The structure of NU-901. For simplicity, hydrogen atoms and benzoate ligands are not shown. (b) Experimental XRD patterns of (i) the as-prepared MOF thin film and (ii) powder scraped from FTO substrates. (iii) Simulated XRD pattern of NU-901.

means of a one-electron oxidation reaction at the TBAPy linkers with the formation of radical cations. The rigid framework prevents the dimerization of radical cations ${ }^{37}$ when the TBAPy linkers are oxidized, resulting in a reversible change in color of the MOF film. The regular and uniform channels of NU-901 should allow ions to migrate through the entire film, yet achieve entry from solution by traversing only short distances. Additionally, the crystal structure of NU-901 features channels that are laterally interconnected by apertures of sufficient size that can permit the ingress and egress of ions such as hexafluorophosphate. Such features result in the remarkable color change and facile switching of the MOF electrochromic thin film.

\section{EXPERIMENTAL SECTION}

Chemicals. All chemicals, benzoic acid (Aldrich, 99.5\%), $\mathrm{ZrCl}_{4}$ (Aldrich, 99.5\%), N,N-dimethylformamide (DMF) (Macron, 99.8\%), dimethylsulfoxide (DMSO) (VWR, 99.9\%), hydrochloric acid (Aldrich, 37\%), acetone (Macron, 98\%), deuterated dimethylsulfoxide (d6-DMSO) (Cambridge, 99\%), deuterated sulfuric acid $\left(\mathrm{D}_{2} \mathrm{SO}_{4}\right)$ (Cambridge, 96-98\% solution in $\mathrm{D}_{2} \mathrm{O}$ ), dichloromethane (DCM) (Sigma-Aldrich, 99.6\%), and tetrabutylammonium hexafluorophosphate $\left(\mathrm{TBAPF}_{6}\right)$ (Fluka, 98.0\%), were used as received without further purification. The chemicals used for the synthesis of the $1,3,6,8$ tetrakis $\left(p\right.$-benzoic acid)pyrene $\left(\mathrm{H}_{4} \mathrm{TBAPy}\right)$ linkers were the same as those reported in our previous work. ${ }^{36 a}$

Preparation of the NU-901 MOF Thin Film. The fluorine-doped tin oxide (FTO) glass substrate ( $15 \Omega /$ sq, Hartford Glass), with a size of $2.5 \times 1.25 \mathrm{~cm}$, was washed in soapy water, ethanol, and acetone by sonication for $15 \mathrm{~min}$ sequentially. Thereafter, the substrate was dried

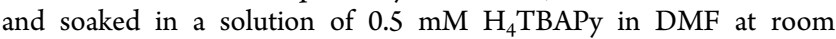
temperature for $12 \mathrm{~h}$. The detailed synthesis of the $\mathrm{H}_{4}$ TBAPy has been reported in our previous work. ${ }^{36 a}$ The substrate was then cleaned with DMF and dried. A 1.35 g portion of benzoic acid and $35 \mathrm{mg}$ of $\mathrm{ZrCl}_{4}$ were added into $8 \mathrm{~mL}$ of DMF in a 6 dram glass vial and ultrasonically dissolved. Thereafter, the solution was placed into an oven at $80{ }^{\circ} \mathrm{C}$ for $2 \mathrm{~h}$. After cooling down the solution to room temperature, $20 \mathrm{mg}$ of $\mathrm{H}_{4} \mathrm{TBAPy}$ was added into this solution and the mixture was sonicated for $20 \mathrm{~min}$ to dissolve all the $\mathrm{H}_{4}$ TBAPy linkers. The solution was then transferred into a $20 \mathrm{~mL}$ screw-thread sample vial (Cole-Parmer, 28 $\mathrm{mm} \times 57 \mathrm{~mm}$ ), equipped with a urea cap and a PTFE foam-backed liner. The as-prepared FTO substrate was then placed into the solution, with the conducting side facing down to the bottom. The cap was closed tightly, and the vial was then placed on the bottom of a gravity convection oven (VWR symphony) with the temperature set at $120{ }^{\circ} \mathrm{C}$. The bottom of the oven provided a temperature gradient inside the vial, which is necessary for the growth of the thin film. The FTO substrate was taken out from the vial after $4.5 \mathrm{~h}$ of reaction. After removing all the precipitations on the back side of the substrate, the substrate was washed with DMF; a uniform yellow MOF thin film could be observed on the front side of the FTO substrate. Finally, the FTO substrate with the MOF thin film was soaked in a pure DMF solution for $24 \mathrm{~h}$ to remove all the excess reagents in the pores of the MOF thin film. After the final step of washing with DMF, the NU-901 MOF thin film was obtained.

Instrumentation. Powder X-ray diffraction (PXRD) experiments were conducted on a Bruker MX $I \mu \mathrm{S}$ microsource with $\mathrm{Cu} \mathrm{K} \alpha$ radiation and an Apex II CCD detector. The samples for PXRD measurements were collected by scraping the as-prepared NU-901 thin films from their substrates. The data were collected on an area detector with rotation frames over $180^{\circ}$ in $\varphi$ and at $2 \theta$ values of 12,24 , and $36^{\circ}$ being exposed for $10 \mathrm{~min}$ at each frame. Overlapping sections of data were matched, and the obtained pattern was integrated using Bruker's APEX2 phase ID program. The PXRD patterns were treated for amorphous background scatter. Thin-film XRD patterns were measured on a Rigaku ATX-G thin-film diffraction workstation. ${ }^{1} \mathrm{H}$ nuclear magnetic resonance (NMR) spectra were recorded on a 500 $\mathrm{MHz}$ Varian INOVA spectrometer and referenced to the residual solvent peak. For NMR sample preparation, the as-prepared NU-901 thin films were scraped from the FTO substrates and dissolved in a few drops of $\mathrm{D}_{2} \mathrm{SO}_{4}$ by sonication. The obtained solution was thus mixed with d6-DMSO and ready for NMR measurement. Diffuse reflectance infrared spectra (DRIFTS) were recorded on a Nicolet 7600 FTIR spectrometer equipped with an MCT detector. The spectra were collected under $\mathrm{N}_{2}$ purge. The samples were prepared by scraping the NU-901 thin films from the FTO substrates and mixing the powder with $\mathrm{KBr}$ in the atmosphere. $\mathrm{KBr}$ was utilized as the background. Scanning electron microscopy (SEM) images were collected on a Hitachi SU8030 SEM, and the transmittance electron microscopy (TEM) images were collected on a Hitachi HT7700 TEM.

Cyclic voltammetry (CV) experiments for the NU-901 thin films were performed on a CHI 660 electrochemical workstation $(\mathrm{CH}$ Instruments, Inc., USA). A three-electrode electrochemical setup was 
used, with the NU-901 thin film/FTO glass electrode, a platinum mesh, and a $\mathrm{Ag} / \mathrm{AgCl} / \mathrm{KCl}$ (sat'd) electrode (homemade) as the working electrode, counter electrode, and reference electrode, respectively. The solution of $0.1 \mathrm{M} \mathrm{TBAPF}_{6}$ in $\mathrm{DCM}$ was used as the electrolyte. The UV-visible (UV-vis) spectra of the NU-901 thin film at various applied potentials were measured on the same electrochemical setup coupled with a Shimadzu 1601 UV-vis spectrometer. The optical transmittance kinetic curve of the NU-901 thin film/FTO glass electrode was also measured on the same setup.

$\mathrm{CV}$ experiments for the $\mathrm{H}_{4}$ TBAPy linker were conducted on the same CHI 660 electrochemical workstation. A platinum disk electrode, a platinum mesh, and a $\mathrm{Ag} / \mathrm{AgCl} / \mathrm{KCl}$ (sat'd) electrode (homemade) were used as the working electrode, counter electrode, and reference electrode, respectively. The DMSO solution containing $0.5 \mathrm{mM}$ of $\mathrm{H}_{4}$ TBAPy and $0.1 \mathrm{M}$ of TBAPF 6 was used as the electrolyte here.

For electron paramagnetic resonance (EPR) measurements, the NU-901 thin films were oxidized by applying $1.6 \mathrm{~V}$ vs $\mathrm{Ag} / \mathrm{AgCl} / \mathrm{KCl}$ (sat'd) on the same electrochemical setup mentioned above in an $\mathrm{Ar}$ glovebox. After $60 \mathrm{~s}$ of applying potential, the blue films on the FTO substrates were removed from the solution and scraped from the substrates. The scraped blue powder was collected into a quartz sample tube ( $2 \mathrm{~mm}$ o.d. $\times 1 \mathrm{~mm}$ i.d.), and then the tube was sealed by UV-curing resin before moving it out from the glovebox. The yellow NU-901 thin films without applying potential were also collected into a quartz tube in the glovebox for reference. Steady-state continuouswave EPR (CW-EPR) spectra were measured using a Bruker Elexsys E580 X-Band EPR spectrometer with a Bruker EN 4118X-MD4 resonator. Microwave power was set to $2 \mathrm{~mW}$, and field modulation was set to $0.010 \mathrm{mT}$ at $100 \mathrm{kHz}$. All measurements were performed at $295 \mathrm{~K}$.

All Raman measurements were conducted using an inverted microscope. The $594 \mathrm{~nm}$ excitation light (spectra-physics, $594 \mathrm{~nm}$ ) was collimated, and a $20 \times$ objective was used to focus laser light onto the sample surface and collect scattered light, which was then dispersed in a $1 / 3 \mathrm{~m}$ spectrograph by a 1200 groove/ $\mathrm{mm}$ grating onto a back-illuminated $\mathrm{LN}_{2}$-cooled charge-coupled device (Spec10:400BR, Princeton Instruments). Raman spectra of the NU-901 thin film at various applied potentials were measured with the same threeelectrode electrochemical setup mentioned above. The solution of 0.1 $\mathrm{M} \mathrm{TBAPF}_{6}$ in DCM was used as the electrolyte. Surface-enhanced Raman spectroscopy (SERS) measurements for the $\mathrm{H}_{4}$ TBAPy ligand were performed on a $\mathrm{Ag}$ film over the nanosphere substrate ${ }^{38}$ fabricated with a sphere mask composed of $390 \mathrm{~nm} \mathrm{SiO}{ }_{2}$ spheres packed onto a piranha cleaned and base-treated \#1.5 glass coverslip. Ag metal $(200 \mathrm{~nm})$ was deposited onto the sphere mask to create a plasmonic substrate for $594 \mathrm{~nm}$ SERS. A $20 \mu \mathrm{L}$ aliquot of the $0.5 \mathrm{mM}$ $\mathrm{H}_{4} \mathrm{TBAPy}$ solution in DMF was dropped on the substrate. After drying in air for $2 \mathrm{~h}$, the substrate was used for SERS measurement.

\section{RESULTS AND DISCUSSION}

Crystallinity of the as-prepared thin films was examined by Xray diffraction (XRD). The XRD patterns of the as-prepared MOF thin film and the powder scraped from the FTO substrate are shown in Figure 1b. Two major diffraction peaks located at 5.3 and $7.6^{\circ}$ can be observed in both experimental patterns, indicating that the polycrystalline MOF film does not show a preferred orientation. Given the nanocrystalline nature of the film, we had to resort to molecular simulations for structure evaluation. ${ }^{16,39}$ As shown in Figure $1 \mathrm{~b}$, both experimental patterns nicely agree with the simulated XRD pattern of NU901. Appling the Scherrer equation to the broad experimental peaks points to a crystallite size of ca. $10 \mathrm{~nm}$; this value is broadly consistent with the rod widths observed by electron microscopy (shown in Figure 2a). The crystal structure of NU901 is composed of diamond-shaped channels with the pore size of $29 \AA$ in length and $12 \AA$ in width; the channel size is large enough for the transport of ions such as tetrabutylammo-
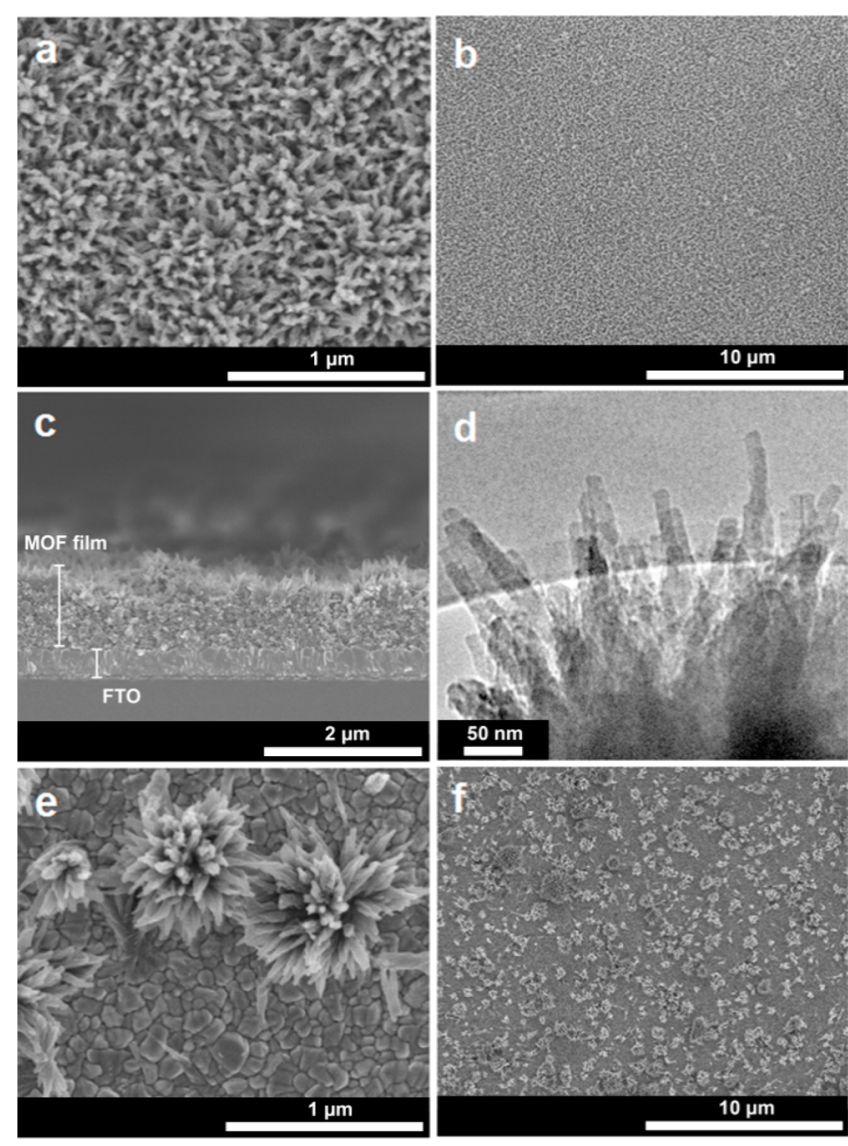

g

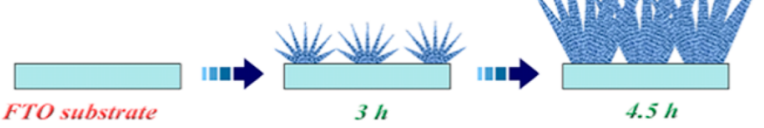

Figure 2. Plane-view SEM images of the NU-901 thin film grown for $4.5 \mathrm{~h}$ at (a) high magnification and (b) low magnification. (c) Crosssectional SEM image of the NU-901 thin film. (d) TEM image of the NU-901 acicular nanorods scraped from the FTO substrate. Planeview SEM images of the NU-901 thin film grown for $3 \mathrm{~h}$ at (e) high magnification and (f) low magnification. (g) Cartoon representation of growth of the NU-901 film.

nium (9.9 $\AA)$ and hexafluorophosphate $(5.5 \AA)$. Note that, in the presence of the substrate and under different synthetic conditions, a completely different MOF topology (with diamond-shaped channels) containing $\mathrm{Zr}_{6}$-based nodes and $\mathrm{H}_{4} \mathrm{TBAPy}$ ligands is formed compared to the MOF with hexagonal channels obtained in our previously reported work. ${ }^{36 a}$ The uniform channels of NU-901 are anticipated to provide ion transport through the whole film, which is beneficial for eliciting electrochromism. ${ }^{1} \mathrm{H}$ nuclear magnetic resonance (NMR) spectra of dissolved samples indicate that the MOF thin film contains both benzoic acid and TBAPy linkers (Figure S1, Supporting Information). This is as expected, given that benzoic acid is known to coordinate to otherwise vacant sites on hexazirconium nodes. ${ }^{36 a}$ The presence of $-\mathrm{OH}$ groups on hexazirconium nodes was confirmed by diffuse reflectance infrared Fourier transform spectroscopy (Figure S2, Supporting Information).

To image the morphology of the NU-901 thin films, planeview scanning electron microscopic (SEM) images were utilized. High- and low-magnification images are shown in 
Figure 2a,b, respectively. It can be observed that the NU-901 thin film is composed of free-standing acicular nanorods, and those nanorods are arranged uniformly on the FTO substrate. Figure $2 \mathrm{~b}$ also shows the uniform, continuous, and crack-free features of the NU-901 thin film in a large region. Crosssectional SEM imaging of the NU-901 film (Figure 2c) reveals a uniform thickness of about $1 \mu \mathrm{m}$, with no gaps between the film and FTO. Figure $2 \mathrm{~d}$ shows a transmission electron microscopic (TEM) image of nanorods of NU-901 scraped from the FTO substrate. From the figure, the widths of the nanorods range from about 10 to $30 \mathrm{~nm}$.

To study the mechanism for the growth of the acicular MOF nanorods on FTO glass, a shorter synthesis period $(3 \mathrm{~h})$ was used. Under these conditions, surface-nucleated microflowers composed of bunches of acicular nanorods were observed (Figure 2e,f). It should be noted that only the bare FTO can be seen between microflowers, implying that nanorods form directly on the electrode rather than on an intervening structureless MOF layer. Finally, with further immersion in the reaction solution, the microflowers continue growing. After $4.5 \mathrm{~h}$, the growing microflowers overlap sufficiently to yield the macroscopically continuous film, composed of micro/nanoscopically of free-standing acicular nanorods, shown in Figure $2 \mathrm{~g}$.

The pretreatment of the FTO substrate before thin film growth (soaking the substrate into the TBAPy solution for 12 h) plays an important role in enabling the mechanism shown in Figure $2 \mathrm{~g}$. The morphologies of the obtained thin films after various syntheses were also recorded when the untreated FTO substrates were directly used for thin film growth (see Figure S3 in the Supporting Information). As shown in Figure S3a, an intervening structureless layer was formed between the FTO substrate and those nanorods.

Cyclic voltammograms (CVs) of an NU-901 thin film on the FTO substrate were measured in a $0.1 \mathrm{M}$ solution of tetrabutylammonium hexafluorophosphate $\left(\mathrm{TBAPF}_{6}\right)$ in dichloromethane (DCM), and the results are shown in Figure 3a. Over the potential range of 0 to $+1.6 \mathrm{~V}$, one redox reaction can be observed, with an anodic peak at $1.56 \mathrm{~V}$ and a cathodic peak at $1.34 \mathrm{~V}$ (vs $\mathrm{Ag} / \mathrm{AgCl} / \mathrm{KCl}$ (sat'd)). The electrochemical oxidation of pyrene-based dendrimers and polypyrene thin films has been reported to happen at similarly positive applied potentials. ${ }^{40,41}$ Because the Zr-nodes in the NU-901 thin film are redox-inactive, the voltammetry peaks observed in Figure 3a may be attributed to redox of the TBAPy linkers. Notably, significant color changes of the NU-901 thin film are observed during CV scans. The CV of the bare FTO substrate was also measured using the same experimental setup (see Figure 3a); the result indicates that the current from the FTO substrate is neglectable compared to that from the NU-901 thin film.

UV-visible (UV-vis) spectra of the NU-901 thin film were measured at various applied potentials; see Figure $3 \mathrm{~b}$. At $0 \mathrm{~V}$, the film shows only a single absorbance maximum (at $405 \mathrm{~nm}$ ); the spectrum is nearly the same at $0.5 \mathrm{~V}$. At $1.2 \mathrm{~V}$, an obvious decrease in the peak at $405 \mathrm{~nm}$ and a slight increase in absorbance at $587 \mathrm{~nm}$ can be seen. Increasing the potential from 1.2 to $1.6 \mathrm{~V}$ yields a large increase in absorbance at 587 $\mathrm{nm}$, as shown in Figure $3 \mathrm{~b}$. These spectroscopic changes are manifest as a change in film color from yellow to deep blue; see Figure $3 \mathrm{c}$. The absorbance of the peak at $405 \mathrm{~nm}$ decreases with increasing applied potential and shows a blue shift in the maximum absorbance. A similar blue shift has been reported for
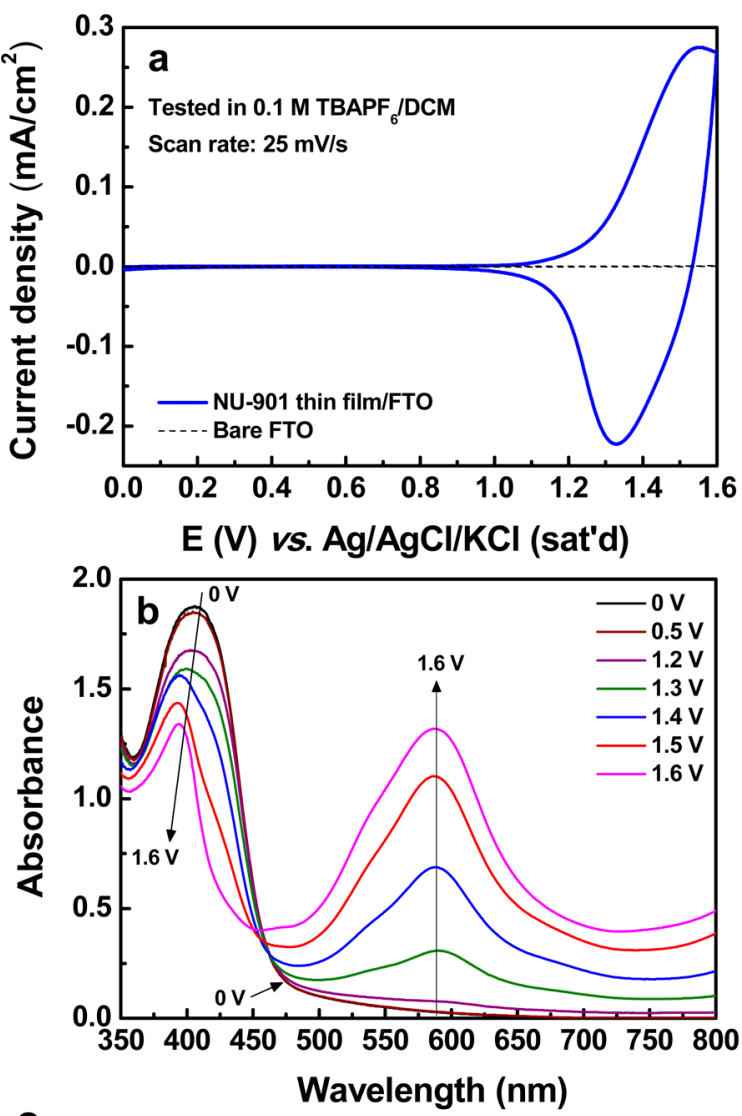

C

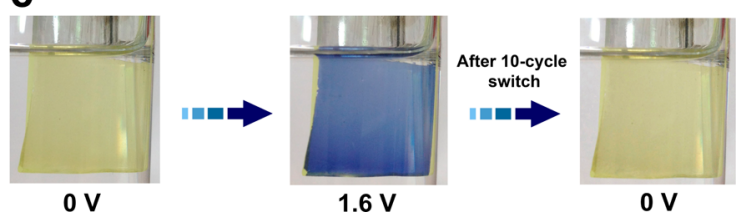

Figure 3. (a) CVs of the NU-901 thin film/FTO electrode and bare FTO electrode. (b) UV-vis spectra of the NU-901 thin film/FTO electrode measured at various applied potentials vs $\mathrm{Ag} / \mathrm{AgCl} / \mathrm{KCl}$ (sat'd). (c) Photos of the NU-901 thin film/FTO electrode at 0 and $1.6 \mathrm{~V}$ vs $\mathrm{Ag} / \mathrm{AgCl} / \mathrm{KCl}$ (sat'd). All experiments were conducted in 0.1 $\mathrm{M} \mathrm{TBAPF} / \mathrm{DCM}$ solution.

a soluble pyrene-based dendrimer upon electrochemical oxidation in DCM. ${ }^{40}$

To assess the reversibility of the film-based redox reaction, electrochromic switching tests between 0 and $1.6 \mathrm{~V}$ were conducted. As shown in Figure 3c, when the potential switches between these limits, a reversible color change from yellow to deep blue is observed. An XRD pattern of NU-901 obtained after 10 cycles of switching from 0 to $1.6 \mathrm{~V}$ is shown in Figure S4 (Supporting Information) and clearly demonstrates that crystallinity is retained.

To understand the chemical basis of the electrochromism, we scraped yellow films (at $0 \mathrm{~V}$ ) and the blue films (at $1.6 \mathrm{~V}$ ) from electrodes in an oxygen-free glovebox and collected continuous-wave electron paramagnetic resonance (CW-EPR) data. Xband spectra are shown in Figure 4a. As expected, the yellow compound yielded no EPR signal. The blue sample (oxidized sample), however, produced a significant signal, indicating the presence of significant radicals. The spectrum agrees with reported CW-EPR spectra of solid-supported pyrene radical cations reported in the literature; ${ }^{42,43}$ thus, NU-901's electrochromism can be attributed to the oxidation of TBAPy linkers. 

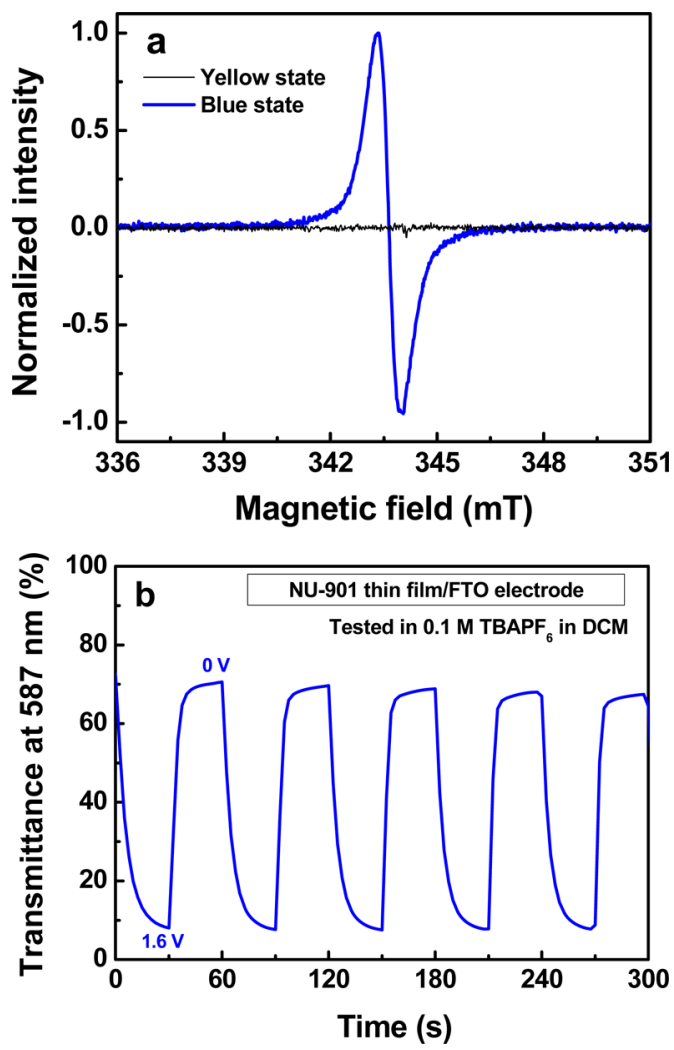

Figure 4. (a) X-Band CW-EPR spectra of the NU-901 samples scraped from the FTO substrates in their yellow state $(0 \mathrm{~V})$ and blue state $(1.6 \mathrm{~V})$. Both spectra were measured at $295 \mathrm{~K}$ with $0.010 \mathrm{mT}$ modulation amplitude. (b) Optical transmittance kinetic curve of the NU-901 thin film/FTO electrode measured at $587 \mathrm{~nm}$ by switching the potential from 0 to $1.6 \mathrm{~V}$ vs $\mathrm{Ag} / \mathrm{AgCl} / \mathrm{KCl}$ (sat'd).

Raman spectroscopy measurements corroborated the assignment. For both the blue and the yellow forms of the film, signals characteristic of pyrene were observed (see Figure S5, Supporting Information). Consistent with pyrene-derived electrochromism, peaks were shifted slightly by film oxidation. Additionally, peaks associated with pyrene were strongly resonantly enhanced for the blue version of the film, as expected if the color change is pyrene-based.

The kinetics of electrochromic switching was followed by recording changes in film transmittance $(587 \mathrm{~nm})$ upon stepping the potential of an NU-901-coated electrode between 0 and $1.6 \mathrm{~V}$ (and vice versa; see Figure $4 \mathrm{~b}$ ). The limiting transmittance change $(\Delta T \%)$ is $62 \%$. The bleaching time and the coloration time, defined as the time to reach $95 \%$ of the limiting change $\Delta T \%$, were found to be 5 and $12 \mathrm{~s}$, respectively. ${ }^{44}$ Additionally, we believe that most of the NU901 thin film is redox-active during the electrochromic switching. ${ }^{45}$ The coloration efficiency of the NU-901 thin film, defined as the absorbance change at $587 \mathrm{~nm}$ between 0 and $1.6 \mathrm{~V}$ divided by the charge passed during the switching, ${ }^{22,46}$ can be determined to be about $204 \mathrm{~cm}^{2} / \mathrm{C}$. The kinetics of electrochromic switching for the MOF film after the removal of benzoates were also investigated to elucidate if the benzoates coordinated on the hexazirconium nodes slow down the observed switching rates (see Figure S7, Supporting Information). Similar bleaching (6 s) and coloration times (10 s) were obtained from the benzoate-free material, indicating that the effect of channel blocking by benzoates on switching rate is negligible. The switching rate of the MOF films may be limited by the transport of TBA ions through the channels of NU-901. Ongoing work within our laboratory is focused on investigating additional electrolytes and developing MOF architectures that will facilitate rapid diffusion. Repetitive CV measurements and long-term electrochromic switching of the NU-901-coated electrode were also investigated, as shown in Figure S8 (Supporting Information). The NU-901 film still exhibits significant redox activity after 20 cycles of CV scan and its $\Delta T \%$ remains $38 \%$ after 60 cycles of electrochromic switching. To investigate the cause for the decreasing $\Delta T \%$, few drops of water were intentionally added into the electrolyte and then the solution was used for the same switching measurement. As shown in Figure S9 (Supporting Information), the $\Delta T \%$ decreases significantly after a few cycles of switching in the wet DCM electrolyte; this observation indicates that the decreasing $\Delta T \%$ in both Figures $\mathrm{S} 8 \mathrm{~b}$ and $\mathrm{S} 9$ (Supporting Information) is attributed to the degradation of pyrene radical cations caused by reaction with water from the environment (open air), which has been reported previously. ${ }^{47}$

$\mathrm{CV}$ measurements were also conducted in a solution of the $\mathrm{H}_{4}$ TBAPy ligand, with a Pt disk as the working electrode. As shown in Figure S10 (Supporting Information), a pyrene oxidation wave is readily observed. The wave, however, is chemically irreversible, presumably because of dimerization of the radical cations ${ }^{37}$ - behavior that is precluded for linkerbased pyrene radicals in the rigid MOF framework.

\section{CONCLUSION}

In summary, a new MOF, NU-901, that can be grown directly on conductive FTO platforms in thin-film form, displays rapid and reversible electrochromic behavior. The electrochromism is facilitated both by the nanoscale porosity of the MOF and by its unusual morphology; NU-901 films are composed of freestanding acicular nanorods, with widths of $10-30 \mathrm{~nm}$. At a thickness of $1 \mu \mathrm{m}$, the films are macroscopically uniform, continuous, and crack-free. Film switching between yellow and deep blue is accomplished by changing the applied potential between 0 and 1.6 V. Resonance Raman and EPR experiments show that the color change is due to one-electron oxidation of pyrene units located at the TBAPy linkers. The robust MOF framework stabilizes the intensely colored pyrene radical cations, in part by spatially isolating them and thereby preventing their dimerization. Ongoing work is focused on modulating the dynamics of electrochromic switching and on accessing other color changes.

\section{ASSOCIATED CONTENT}

\section{S Supporting Information}

NMR spectrum, DRIFTS spectrum, Raman spectra, long-term electrochromic switching data, CV curve for TBAPy ligand, and the discussion about removal of benzoates form the MOF films are given in the Supporting Information (PDF). This material is available free of charge via the Internet at http://pubs.acs.org.

\section{AUTHOR INFORMATION}

\section{Corresponding Authors}

*E-mail: o-farha@northwestern.edu (O.K.F.).

*E-mail: j-hupp@northwestern.edu (J.T.H.).

\section{Notes}

The authors declare no competing financial interest. 


\section{ACKNOWLEDGMENTS}

O.K.F. and J.T.H. gratefully acknowledge support from the U.S. Dept. of Energy, Office of Science, Basic Energy Sciences (grant no. DE-FG02-87ER13808), and Northwestern University. M.R.W. was supported by the Chemical Sciences, Geosciences, and Biosciences Division, Office of Basic Energy Sciences, DOE under grant no. DE-FG02-99ER14999. W.B. acknowledges support from the Foundation for Polish Science through the "Kolumb" Program. J.E.M. acknowledges a DOE EERE Postdoctoral Research Award, EERE Fuel Cell Technologies Program, administered by ORISE for DOE. ORISE is managed by ORAU under DOE Contract DE-AC05-060R23100. C.W.K. acknowledges support from the Graduate Students Study Abroad Program sponsored by the National Science Council (Taiwan). D.F.-J. acknowledges the Royal Society (U.K.) for a University Research Fellowship. D.M.G. was supported by the Department of Defense through the National Defense Science \& Engineering Graduate (NDSEG) Fellowship Program. J.C.B. is supported by the NDSEG Fellowship (32 CFR 168a) from the Department of Defense and also gratefully acknowledges support of a Ryan Fellowship from the NU International Institute for Nanotechnology (IIN).

\section{REFERENCES}

(1) Rosseinsky, D. R.; Mortimer, R. J. Adv. Mater. 2001, 13, 783.

(2) Heuer, H. W.; Wehrmann, R.; Kirchmeyer, S. Adv. Funct. Mater. 2002, 12, 89.

(3) Mortimer, R. J.; Dyer, A. L.; Reynolds, J. R. Displays 2006, 27, 2. (4) Baetens, R.; Jelle, B. P.; Gustavsen, A. Sol. Energy Mater. Sol. Cells 2010, 94, 87.

(5) Mortimer, R. J. Annu. Rev. Mater. Res. 2011, 41, 241.

(6) Santato, C.; Odziemkowski, M.; Ulmann, M.; Augustynski, J. J. Am. Chem. Soc. 2001, 123, 10639.

(7) Niklasson, G. A.; Granquist, C. G. J. Mater. Chem. 2007, 17, 127.

(8) Itaya, K.; Shibayama, K.; Akahoshi, H.; Toshima, S. J. Appl. Phys. 1982, 53, 804

(9) Baioni, A. P.; Vidotti, M.; Fiorito, P. A.; Ponzio, E. A.; de Torresi, S. I. C. Langmuir 2007, 23, 6796.

(10) Lin, T. H.; Ho, K. C. Sol. Energy Mater. Sol. Cells 2006, 90, 506.

(11) Tseng, C. Y.; Hu, C. W.; Huang, K. C.; Chang, L. C.; Vittal, R.; Ho, K. C. Electrochim. Acta 2013, 101, 232.

(12) Furukawa, H.; Cordova, K. E.; O’Keeffe, M.; Yaghi, O. M. Science 2013, 341, 1230444.

(13) Férey, G. Chem. Soc. Rev. 2008, 37, 191.

(14) Meek, S. T.; Greathouse, J. A.; Allendorf, M. D. Adv. Mater. 2011, 23, 249.

(15) Farha, O. K.; Eryazici, I.; Jeong, N. C.; Hauser, B. G.; Wilmer, C. E.; Sarjeant, A. A.; Snurr, R. Q.; Nguyen, S. T.; Yazaydin, A. O.; Hupp, J. T. J. Am. Chem. Soc. 2012, 134, 15016.

(16) Farha, O. K.; Yazaydin, A. O.; Eryazici, I.; Malliakas, C. D.; Hauser, B. G.; Kanatzidis, M. G.; Nguyen, S. T.; Snurr, R. Q.; Hupp, J. T. Nat. Chem. 2010, 2, 944.

(17) Li, J. R.; Sculley, J.; Zhou, H. C. Chem. Rev. 2012, 112, 869.

(18) Ma, L. Q.; Abney, C.; Lin, W. B. Chem. Soc. Rev. 2009, 38, 1248.

(19) Lee, J.; Farha, O. K.; Roberts, J.; Scheidt, K. A.; Nguyen, S. T.;

Hupp, J. T. Chem. Soc. Rev. 2009, 38, 1450.

(20) Kreno, L. E.; Leong, K.; Farha, O. K.; Allendorf, M.; Van Duyne,

R. P.; Hupp, J. T. Chem. Rev. 2012, 112, 1105.

(21) Noh, C. H.; Jeon, S. J.; Son, S. U. U. S. Patent US 2011/ $0235151 \mathrm{~A} 1$.

(22) Wade, C. R.; Li, M.; Dincă, M. Angew. Chem., Int. Ed. 2013, 52, 13377.

(23) Burda, C.; Chen, X.; Narayanan, R.; El-Sayed, M. A. Chem. Rev. 2005, 105, 1025.

(24) Xia, Y. N.; Yang, P. D.; Sun, Y. G.; Wu, Y. Y.; Mayers, B.; Gates, B.; Yin, Y. D.; Kim, F.; Yan, Y. Q. Adv. Mater. 2003, 125, 353.
(25) Liu, K.; You, H. P.; Zheng, Y. H.; Jia, G.; Zhang, L. H.; Huang, Y. J.; Yang, M.; Song, Y. H.; Zhang, H. J. CrystEngComm 2009, 11, 2622.

(26) Jahan, M.; Bao, Q. L.; Yang, J. X.; Loh, K. P. J. Am. Chem. Soc. 2010, 132, 14487.

(27) Li, R.; Yuan, Y. P.; Qiu, L. G.; Zhang, W.; Zhu, J. F. Small 2012, $8,225$.

(28) Rieter, W. J.; Taylor, K. M. L.; An, H. Y.; Lin, W. L.; Lin, W. B. J. Am. Chem. Soc. 2006, 128, 9024.

(29) Giri, S.; Saha, S. K. J. Phys. Chem. C 2010, 114, 11723.

(30) Bataille, T.; Bracco, S.; Comotti, A.; Costantino, F.; Guerri, A.; Ienco, A.; Marmottini, F. CrystEngComm 2012, 14, 7170.

(31) Karimi, Z.; Morsali, A. J. Mater. Chem. A 2013, 1, 3047.

(32) Li, Y. S.; Bux, H.; Feldhoff, A.; Li, G. L.; Yang, W. S.; Caro, J. Adv. Mater. 2010, 22, 3322.

(33) Zhuang, J. L.; Lommel, K.; Ceglarek, D.; Andrusenko, I.; Kolb, U.; Maracke, S.; Sazama, U.; Froba, M.; Terfort, A. Chem. Mater. 2011, 23, 5366.

(34) Mao, Y. Y.; Huang, H. B.; Liu, Y.; Shi, L.; Cao, W.; Li, J. W.; Sun, L. W.; Peng, X. S. CrystEngComm 2013, 15, 5591.

(35) Rabin, O.; Herz, P. R.; Lin, Y. M.; Akinwande, A. I.; Cronin, S. B.; Dresselhaus, M. S. Adv. Funct. Mater. 2003, 13, 631.

(36) The use of $\mathrm{H}_{4}$ TBAPy as a MOF linker has been previously reported: (a) Mondloch, J. E.; Bury, W.; Fairen-Jimenez, D.; Kwon, S.; Demarco, E. J.; Weston, M. H.; Sarjeant, A. A.; Nguyen, S. T.; Stair, P. C.; Snurr, R. Q.; Farha, O. K.; Hupp, J. T. J. Am. Chem. Soc. 2013, 135, 10294. (b) Stylianou, K. C.; Heck, R.; Chong, S. Y.; Bacsa, J.; Jones, J. T. A.; Khimyak, Y. Z.; Bradshaw, D.; Rosseinsky, M. J. J. Am. Chem. Soc. 2010, 132, 4119. (c) Stylianou, K. C.; Rabone, J.; Chong, S. Y.; Heck, R.; Armstrong, J.; Wiper, P. V.; Jelfs, K. E.; Zlatogorsky, S.; Bacsa, J.; McLennan, A. G.; Ireland, C. P.; Khimyak, Y. Z.; Thomas, K. M.; Bradshaw, D.; Rosseinsky, M. J. J. Am. Chem. Soc. 2012, 134, 20466.

(37) Hara, M.; Tojo, S.; Kawai, K.; Majima, T. Phys. Chem. Chem. Phys. 2004, 6, 3215.

(38) Hulteen, J. C.; Treichel, D. A.; Smith, M. T.; Duval, M. L.; Jensen, T. R.; Van Duyne, R. P. J. Phys. Chem. B 1999, 103, 3854.

(39) Fairen-Jimenez, D.; Colon, Y. J.; Farha, O. K.; Bae, Y. -S.; Hupp, J. T.; Snurr, R. Q. Chem. Commun. 2012, 48, 10496.

(40) Gingras, M.; Placide, V.; Raimundo, J.-M.; Bergamini, G.; Ceroni, P.; Balzani, V. Chem.-Eur. J. 2008, 14, 10357.

(41) Xu, L. Y.; Zhao, J. S.; Liu, R. M.; Liu, H. T.; Liu, J. F.; Wang, H. S. Electrochim. Acta 2010, 55, 8855.

(42) Mao, Y.; Thomas, J. K. Langmuir 1992, 8, 2501.

(43) Mao, Y.; Thomas, J. K. J. Phys. Chem. 1995, 99, 2048.

(44) A video recorded during switching between 0 and $1.6 \mathrm{~V}$ is included in the Supporting Information.

(45) The current recorded during the electrochromic switching is shown in Figure S6 (Supporting Information). The charge passed during the color change is estimated to be $4.7 \mathrm{mC} / \mathrm{cm}^{2}$. The active film mass used for color change can be calculated to be $65 \mu \mathrm{g} / \mathrm{cm}^{2}$ (molecular weight of the repeated unit of NU-901 containing two TBAPy linkers and one node is 2653). This value is close to the film mass estimated by scraping films and weighing (about $0.2-0.3 \mathrm{mg}$ per $3.125 \mathrm{~cm}^{2}$ sample), implying that majority of the NU-901 thin film is redox-active during the electrochromic switching.

(46) Gaupp, C. L.; Welsh, D. M.; Rauh, R. D.; Reynolds, J. R. Chem. Mater. 2002, 14, 3964.

(47) Oyama, M.; Matsui, J. J. Electroanal. Chem. 2004, 570, 77. 